\title{
L'éloquence des cartes.De la défense du territoire national à la bataille de Bagdad
}

Maps' eloquence. From national territory's defence to Baghdad battle

La elocuencia del mapa. De la defensa del territorio nacional a la batalla de

Bagdad

Emmanuelle Bonerandi et Myriam Houssay-Holzschuch

\section{OpenEdition}

\section{Journals}

Édition électronique

URL : https://journals.openedition.org/mots/16162

DOI : $10.4000 /$ mots. 16162

ISSN : 1960-6001

Éditeur

ENS Éditions

Édition imprimée

Date de publication : 1 novembre 2003

Pagination : 73-85

ISBN : 2-84788-043-7

ISSN : 0243-6450

Référence électronique

Emmanuelle Bonerandi et Myriam Houssay-Holzschuch, «L'éloquence des cartes.De la défense du territoire national à la bataille de Bagdad », Mots. Les langages du politique [En ligne], 73 | 2003, mis en ligne le 09 octobre 2008, consulté le 24 avril 2022. URL : http://journals.openedition.org/mots/16162 ; DOI : https://doi.org/10.4000/mots.16162 


\section{L'éloquence des cartes. De la défense du territoire national à la bataille de Bagdad}

Beaucoup plus qu'une série de statistiques ou qu'un ensemble d'écrits, la carte est la forme de représentation géographique par excellence; c'est sur la carte que doivent être portés tous les renseignements nécessaires à l'élaboration des tactiques et des stratégies.

Yves Lacoste, 1976

L'élaboration d'une campagne militaire implique une parfaite connaissance préalable du terrain. Une campagne mal préparée peut conduire au fiasco. Une méconnaissance de la topographie locale, des variations climatiques (pluies torrentielles ininterrompues de mousson en Asie du Sud-Est, chaleurs insupportables dès le printemps dans le désert), et l'opération, qui devait se dérouler en un éclair, piétine, s'enlise, voire recule. Les termes géographiques sont indissociables du vocabulaire belliqueux: terrain, glacis, champ, percée, verrou, crête, etc. Pour un peu, on déclarerait la géographie fille ainée de la guerre, pour relancer la polémique autour de La géographie, ça sert, d'abord, à faire la guerre, ouvrage publié en 1976 par le géographe Yves Lacoste.

C'est sans doute à travers les relations entretenues entre la représentation cartographique et la guerre que l'on perçoit le mieux "l'éloquence des cartes $»^{2}$. Nous considérons ici la guerre dans un sens très large, qui renvoie évidemment aux combats armés dans le cadre d'un conflit, mais également

1. UMR Géographie-cités/Géophile, ENS-LSH - 15, Parvis René-Descartes BP 7000 69342 Lyon cedex - ebonerandi@ens-lsh.fr - Myriam.Houssay-Holzschuch@ens-1sh.fr.

2. L'expression est empruntée à Carl Sauer, géographe culturel américain. 
en amont à la sécurité des territoires dans le cadre d'une défense passive, et plus généralement à la connaissance fournie aux États par les militaires et les services de renseignement. La carte est alors à envisager comme une forme de savoir socialement construite: elle reflète l'idéologie et la vision du monde de ses producteurs. C'est dans ce sens que l'on peut considérer l'apport majeur de la géographie. En temps de paix, la carte contient des données stratégiques pour la sécurité nationale et peut à ce titre être sujette à la censure. Enfin, la carte sert à la connaissance et donc aussi au contrôle de l'espace : les techniques géographiques qu'elle emploie soutiennent et promeuvent les opérations militaires.

\section{Les géographes et l'interprétation des cartes}

L'apport critique des géographes a essentiellement porté sur deux points : l'analyse des distorsions délibérées du contenu des cartes et une réflexion sur la nature discursive de la carte. Selon le géographe américain Brian Harley, la carte constitue une forme construite, donc manipulée, du savoir, renvoyant à l'association entre savoir et pouvoir. La carte peut être considérée comme une image liée à un contexte historique précis et chargée d'un jugement de valeur $^{3}$. La carte peut être assimilée à une forme de langage. Le discours cartographique porte sur les aspects d'un texte (ici la carte) qui ont une portée appréciative, évaluative, persuasive ou rhétorique, par opposition à ceux qui traitent seulement de la dénomination, de la localisation et de la narration. Le discours passe autant dans les silences (omissions) que dans les éléments mis en valeur (déformés).

Mark Monmonier ${ }^{4}$ insiste sur la portée symbolique des cartes. Il compare leur fonctionnement à de véritables « icônes » politiques, symboles du pouvoir, de l'autorité et de l'unité nationale. C'est le rôle joué par la plupart des atlas nationaux, comme message subliminal ou avéré. Les planches consacrées à la formation du territoire national en sont une illustration, notamment

3. «Par la sélectivité de leur contenu et par leurs symboles et leurs styles de représentation, les cartes sont un moyen d'imaginer, d'articuler et de structurer le monde des hommes » (B. Harley, 1988, « Maps, knowledge and power » dans The Iconography of Landscape : Essays on the Symbolic Representation, Design and Use of Past Environnements, publié sous la direction de Denis Cosgrove et Stephen Daniels, New York, Cambridge University Press, traduit en français dans Gould P., Bailly A., [ed.], 1995, Le pouvoir des cartes - Brian Harley et la cartographie, Paris, Anthropos).

4. M. Monmonier, 1993, chap. 7 : «Les cartes au service de la propagande politique », p. 133-176. 
dans les États de création récente. Pensons simplement à l'Italie et à l'Allemagne. Bien entendu, les États indépendants postcoloniaux renvoient à l'utilisation de cartes par les anciennes puissances coloniales pour légitimer leurs conquêtes territoriales, leur exploitation économique et leur impérialisme culturel, rappelant l'aphorisme de la plume plus puissante que l'épée. Certains États vont même jusqu'à se livrer une guerre que l'on pourrait définir comme cartographique lorsque des territoires contestés sont représentés comme acquis à l'aide d'une simple annexion graphique à l'intérieur des frontières nationales. On peut citer l'exemple de l'État disputé du Jammu-etCachemire à la frontière de l'Inde, du Pakistan et de la Chine, chaque pays incluant sur le papier la région dans les limites de son autorité nationale.

Les géographes ont également largement étudié le rôle déformant des systèmes de projection (figure 1) pour augmenter la taille et l'importance d'un pays ou, au contraire les réduire pour le faire apparaitre comme menac .
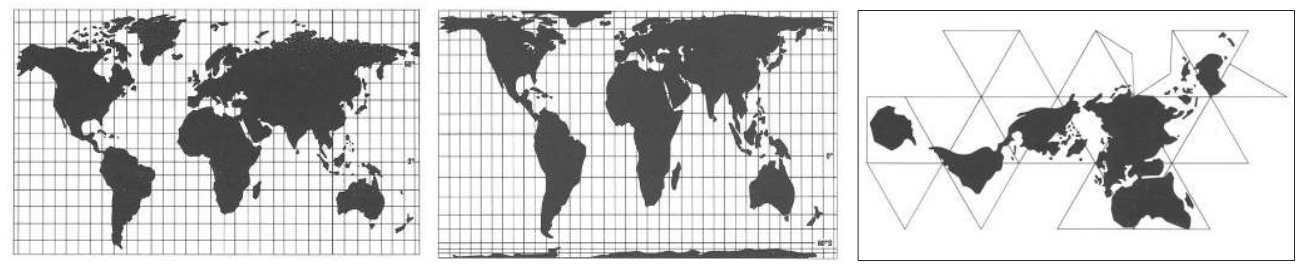

Figure 1

Des projections qui changent la face du monde (sources : Brunet, Dollfus, 1994 ; Brunet R., Ferras R., Théry H., 1992). La projection de Mercator (à gauche) est une projection conforme qui conserve les angles. Méridiens et parallèles s'y coupent à angle droit ; elle étend exagérément les espaces des hautes latitudes. La projection de Peters (au centre) est une projection équivalente; elle conserve les surfaces mais déforme les contours. La projection de Fuller (à droite) donne les bonnes formes et les bonnes proportions, au prix d'une vision éclatée du monde.

Ainsi, le planisphère élaboré à partir de la projection de Mercator établie au 16e siècle fut utilisé à la fois pour flatter l'Empire britannique placé au centre du monde, pour valoriser les démocraties industrielles de l'hémisphère Nord, et pour cerner la menace communiste de la Chine et de l'URSS coloriées en rouge vif. À cette projection largement diffusée, répond, au début des années 1970, celle de Peters établie à égalité de surface qui fut adoptée par l'Unesco. Mais à l'échelle mondiale, on semble s'éloigner du sujet...

L'Allemagne nazie fournit un exemple de choix d'État qui eut largement recours à la carte comme outil de propagande au service de son idéologie. À 
l'aide des cartes de l'hebdomadaire Facts in Review publié à New York par la «German Library of Information » de 1939 à 1941, la propagande nazie entend justifier auprès des lecteurs d'outre-Atlantique la nécessité de l'expansion allemande et obtenir le maintien de la neutralité américaine. Ainsi la couverture du numéro du 8 décembre 1939 propose deux cartes qui comparent la situation de l'Allemagne en 1914 et en 1939 et renvoient à la thématique de l'encerclement. La situation à la veille de la seconde guerre mondiale offre la vision d'une Allemagne bien éloignée de sa réelle expansion territoriale. Ainsi même si l'État nazi n'ose revenir sur la perte de la partie occidentale de la Pologne, il ne signale pas la remilitarisation de la Rhénanie, le retour de la Sarre dans son giron suite au plébiscite, de même que l'Anschluss de l'Autriche, ou l'annexion des Sudètes en 1938. Afin de renforcer la démonstration, la carte définit l'Italie et l'URSS comme des pays à la neutralité bienveillante et omet de les présenter comme des puissances de l'axe. Tout aussi édifiante et persuasive est cette «Étude des empires 》 publiée en février 1940 (figure 2) qui met en parallèle les $684535 \mathrm{~km}^{2}$ et les 87 millions

\section{A STUDY IN EMPIRES}

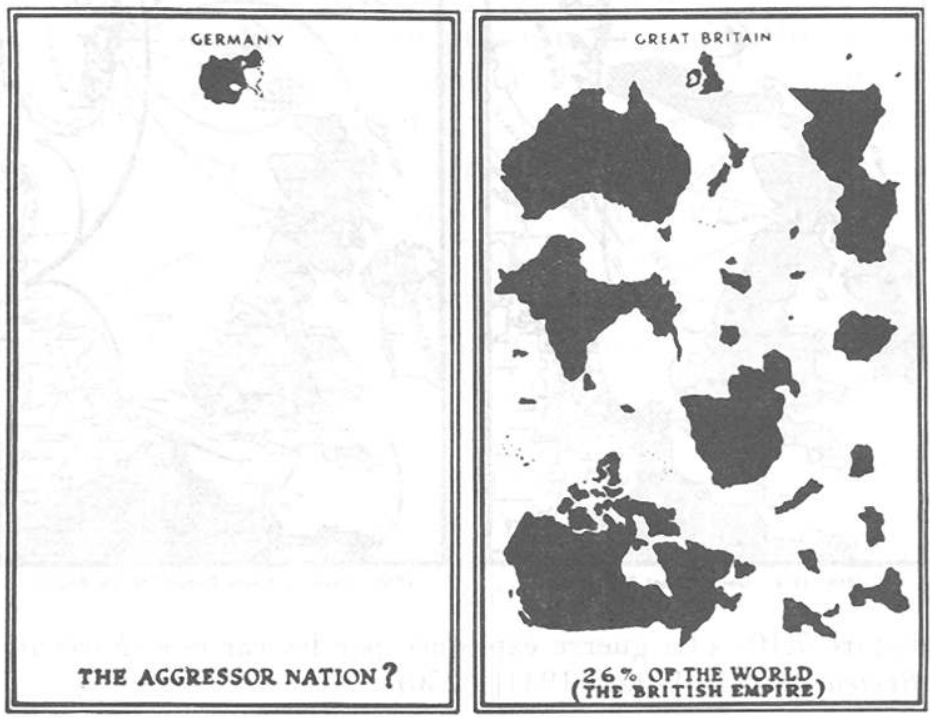

Figure 2

Exemple d'utilisation de la carte par la propagande nazie (source : Monmonier M., 1993) 
d'habitants de l'Allemagne avec les $34500000 \mathrm{~km}^{2}$ conquis par l'Angleterre, soit $26 \%$ de la surface des terres émergées du globe pour seulement 46 millions d'habitants. Comment la petite Allemagne pourrait-elle être agresseur? Ne s'agit-il pas là d'une éloquente leçon de cartes pour légitimer une guerre mondiale?

\section{Carte et sécurité nationale}

Selon M. Monmonier, la carte représente une arme tactique et stratégique fondamentale. Il semble donc assez naturel que les gouvernements protègent leurs cartes, mais peut-être moins qu'ils maquillent les informations géographiques et diffusent parfois des cartes volontairement falsifiées, la carte pouvant devenir instrument de propagande politique. D'après B. Harley, les gouvernements pratiquent deux formes de censure : la censure du secret pour servir la défense militaire, et la censure du silence pour imposer ou renforcer les valeurs nationales.

On conçoit aisément qu'il existe un besoin réel de sécurité cartographique et que les gouvernements cherchent à protéger leurs données. "Science des princes », la cartographie apporte des connaissances, et donc de la puissance, sur le territoire. Si cette connaissance passe à l'ennemi, elle devient menace, à moins qu'on ne la falsifie. Ainsi les maquettes en trois dimensions des villes fortifiées françaises établies par les généraux à la demande de Louis XIV en vue de la planification des batailles, et présentées aujourd'hui au musée des Plans-Reliefs de l'Hôtel des Invalides, ont été protégées comme des secrets d'État et classées "secret défense » jusqu'à la fin de la Seconde Guerre mondiale. L'édification de la couverture cartographique du territoire français révèle également cette culture du secret entretenue par l'armée ${ }^{5}$. En 1747, Cassini III se voit confier par Louis XV la réalisation d'une carte à la fois "générale » (qui couvre toute la France) et "particulière » (puisqu'elle est au $1 / 86400^{\mathrm{e}}$, échelle réservée aux cartes régionales). L'administration royale a besoin de disposer de cartes précises pour une gestion plus rigoureuse de son territoire. La défense des côtes bretonnes exige, notamment, une planification de leur surveillance. La carte de Cassini est réalisée selon un découpage géométrique. La projection triangulaire défendue par l'Académie des Sciences assure un positionnement rigoureux des points, l'armée (le Génie) le rendu topographique, notamment dans les zones de montagne. En 1756, face aux difficultés de financement, l'opération est privatisée, les rap-

5. Voir M. Pelletier, 1990. 
ports entretenus sur le terrain entre les ingénieurs de Cassini et l'armée deviennent difficiles. La méfiance, voire l'hostilité, des militaires est d'autant plus grande lorsqu'il s'agit des régions frontières. Ainsi, à propos des cartes du Dauphiné et de la Provence réalisées dans les années 1770, le major du génie Darçon écrit :

[...] ce qui importe beaucoup, c'est que les chaines de montagnes, qui ne présentent que des passages déterminés sur une frontière hérissée d'obstacles, pouvant réellement tenir lieu de fortifications, il est essentiel de n'en indiquer ni le fort ni le faible à l'ennemi, et il est de la plus grande importance de n'en rendre la connaissance profitable que pour nous. Le privilège accordé aux ingénieurs de $\mathrm{M}$. de Cassini devrait excepter les parties de frontières dont il serait important de réserver la connaissance... Sa carte sera bonne ou mauvaise. Si elle était bonne, il faudrait l'interdire $[\ldots]$.

Confisquées et nationalisées sous la Révolution, les cartes de Cassini sont, à partir de 1793, stockées au dépôt de la Guerre, dont dépendent les ingénieurs géographes militaires. L'époque révolutionnaire voit l'achèvement de la carte de Cassini. Deux types d'utilisateurs se font alors face : d'une part, la nouvelle administration, d'autre part les militaires. Ces derniers assurent la mise à jour des voies de communication, mais, jusqu'en 1815, ils empêchent la diffusion au nom de la sécurité nationale. En 1817, est formée la commission chargée « d'examiner le projet d'une nouvelle carte topographique générale de la France, appropriée à tous les services publics, et combinée avec le cadastre général ». Ces nouvelles feuilles au 1/80 000 , publiées de 1832 à 1880, élaborées par le service géographique de l'armée, portent alors le nom de cartes d'état-major. Le décret du 27 juin 1940 supprime ce service et crée l'Institut géographique national sur la proposition du maréchal de France, Président du conseil, et des ministres, entre autres, de la défense nationale et de la guerre.

En terme de falsification volontaire, les cartes soviétiques représentent des exemples largement connus. À la fin des années 1930, les services cartographiques passent sous le contrôle du NKVD qui s'emploie à modifier systématiquement la forme et la position des villages, des côtes, des fleuves, des infrastructures de transport, des bâtiments, des frontières dans les atlas destinés à l'usage public. Le but recherché est de tromper les adversaires potentiels dans l'établissement des cartes pour le guidage des "missiles de croisière ». Ces déformations connaissent leur apogée vers le milieu des années 1960, au moment où les États-Unis commencent à déployer une couverture de satellites-espions. Obsédés par le syndrome de l'attaque, les Soviétiques publient les cartes touristiques des aires urbaines sans échelle, sans les principaux axes de circulation, allant jusqu'à omettre la présence massive du 
siège du KGB sur la place Djerzinsky. Il est évident que la falsification s'est largement périmée avec les perfectionnements des systèmes d'analyse numérique des images et la souplesse de trajectoire des satellites militaires qui offrent des résolutions permettant de lire jusqu'au numéro d'immatriculation des véhicules.

À partir des années 1970, les gouvernements prennent en compte les progrès technologiques. Les archives sont informatisées et stockées sur des cartes électroniques. Mais comme elles sont vulnérables aux piratages informatiques par virus ou à la radiation électromagnétique, on a encore recours à des copies de sauvegarde sur papier.

Enfin, il ne faudrait pas considérer la censure du silence comme le seul fait des régimes dictatoriaux. L'usage est courant dans la plupart des démocraties occidentales. Ainsi, l'Ordnance Survey, agence cartographique nationale anglaise, a établi une liste des sites «sensibles » omis ou maquillés sur les cartes et photographies aériennes, les cartes grecques présentent des zones totalement blanches en Épire, à la frontière sensible entre la Grèce et l'Albanie, de même que l'Institut géographique national de notre pays recouvre les principaux sites nucléaires d'un très significatif voile blanc.

\section{Connaitre le terrain : techniques géographiques et opérations militaires}

Les géographes ne possèdent pas le monopole de la représentation cartographique, loin s'en faut. Bien avant le développement des nouvelles technologies, les militaires étaient déjà à la pointe de la représentation cartographique des territoires nationaux : il n'est que de penser aux cartes d'étatmajor réalisées en France à partir de 1832. Indéniablement, les militaires possèdent une maitrise technique qui leur assure une bonne connaissance du terrain. La carte constitue un rouage essentiel dans l'infrastructure technique de l'armée sur le terrain. On peut définir deux types de cartes propres à l'usage militaire dans un contexte de conflit armé : la carte de connaissances du terrain (savoir topographique brut des cartes), et la carte d'avancées des troupes (de bataille).

Bien entendu, ces cartes, du fait de leur usage militaire et de leurs fonctions défensives ou offensives, sont gardées au secret. Connaitre le plan de déploiement des troupes dans Paris en cas d'insurrection serait bien entendu voué à l'échec, même si nombre de civils savent que ce type de documents existe. Ainsi, pour ce qui concerne la France, le chercheur ne peut consulter les cartes militaires au Service historique des armées de Vincennes qu'une 
fois que les archives sont ouvertes et accessibles. Si le délai ordinaire de communication des archives publiques est de trente ans à compter de la date de l'acte ou de la date de clôture du dossier, un certain nombre de documents sont soumis à des délais spéciaux $(60,100,120$ ou 150 ans) qui les rendent non communicables.

Cependant, la société de l'information rend régulièrement compte de conflits armés. Pour cela, les médias ont recours à des cartes qui ressemblent fort étrangement aux cartes militaires, qu'il s'agisse des cartes de connaissance du terrain ou des cartes d'avancées des troupes et des combats, et ce dans un souci que l'on pourrait qualifier de réalisme et d'immédiateté, mais aussi de diffusion d'une information (re)construite dans un but hautement persuasif. Lorsque le conflit met en scène les troupes nationales, l'enjeu politique est évident et les médias du pays concerné présentent un point de vue partisan, relayant ou dénonçant les informations fournies par les services de presse des armées. La plupart du temps, le lecteur critique ne peut accéder à la source originelle de la carte.

La guerre entamée sur le terrain irakien par les États-Unis et la GrandeBretagne le 20 mars 2003 offre un florilège de représentations cartographiques. Quel média n'a pas diffusé les cartes d'avancée des troupes pour étayer son discours, qu'il s'agisse de la fulgurante course dans le désert des premiers jours $^{6}$, du doute de la deuxième semaine ou de "l'ultime» bataille de Bagdad? Dès les premières frappes, les sites internet de ces mêmes médias ont fourni des cartes interactives pour donner l'impression au lecteur de suivre la bataille en temps réel aux côtés des GI's. On peut, à partir de ces documents, se livrer à une analyse assez édifiante du discours performatif mis en place par les chefs d'état-major coalisés, et largement relayés par les médias outre-Atlantique mais aussi européens, et français ${ }^{7}$. Nous nous sommes intéressées aux cartes diffusées par deux médias américains de grande diffusion, l'un de presse (USA Today, quotidien populaire comparable au Parisien), l'autre de télévision $(\mathrm{CNN})^{8}$. Cet exemple montre la difficulté à connaitre l'origine des cartes diffusées, aussi bien pour CNN que pour USA Today. On

6. Parfois même assimilée à un « Paris-Dakar bis ».

7. Il est assez difficile d'établir une distinction nationale claire entre médias, tant les cartes, notamment celles de l'avancée des troupes coalisées, sont semblables de part et d'autre de l'Atlantique. Même si la démonstration s'appuie sur l'utilisation de cartes établies par des médias américains, on ne peut véritablement distinguer dans le discours cartographique un camp de la paix d'un camp de la guerre dans les quotidiens et les hebdomadaires.

8. http://www.usatoday.com/news/world/iraq/front.htm\#; http://www.cnn.com/SPE CIALS/2003/iraq/maps/ 
ne peut savoir si les cartes en ligne sont des cartes conçues directement par les journalistes, ou des cartes militaires fournies aux journaux lors des conférences de presse, ou même des cartes réalisées à partir d'informations fournies par les services secrets des pays concernés. Aucune notice explicative n'est fournie avec le document qui s'affiche à l'écran. L'éthique journalistique garantit à elle seule l'information transmise. Pour le chercheur, un paradoxe apparait entre le réalisme voulu des cartes et l'opacité des sources.

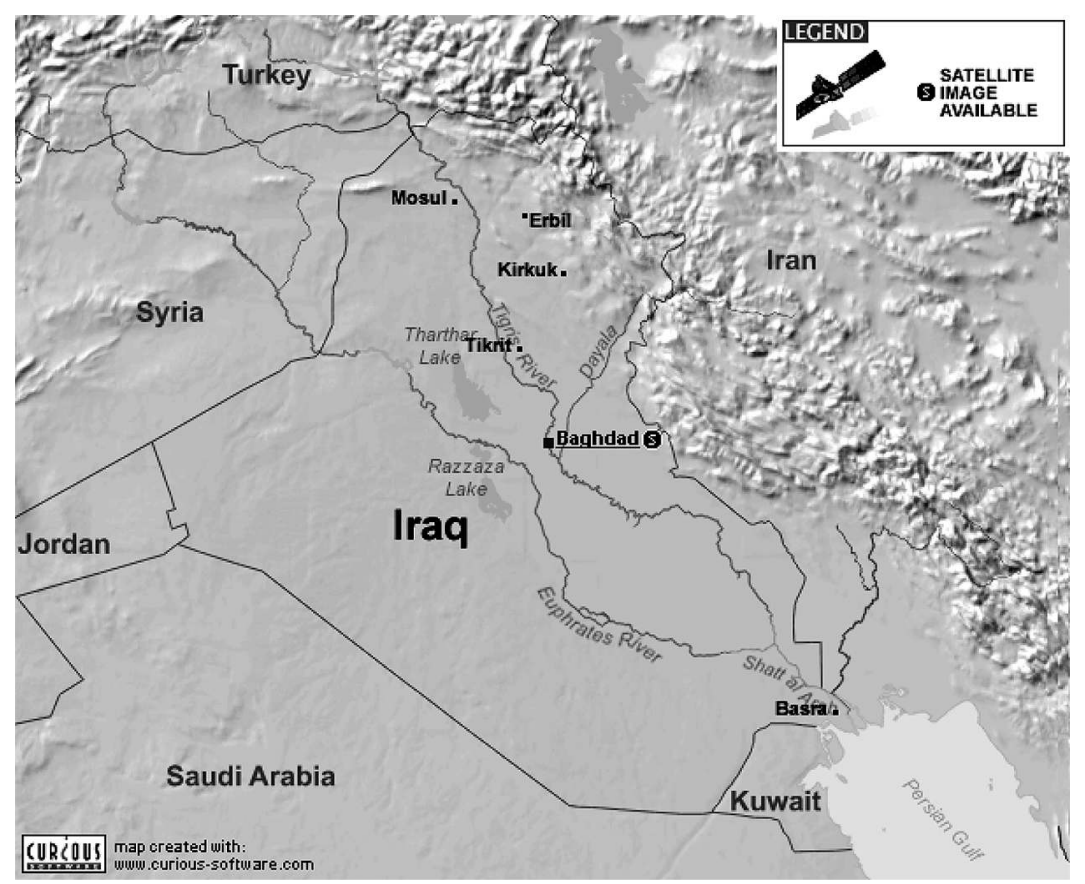

Figure 3

Le terrain irakien vu par CNN, avril 2003

Le site internet de la chaine CNN propose une série de cartes de situation que l'on peut classer comme cartes de connaissance du terrain. On y trouve en première ligne la carte du terrain irakien (figure 3), carte de facture classique présentant le relief de la région, le réseau hydrographique et les principales villes sur un fond de carte politique (figuré des frontières nationales). Cette carte est accompagnée du sobre commentaire suivant :

Iraq, at 168,927 square miles (437,521 square kilometers), is slightly larger than California. The land is mostly broad desert plains but has reedy marshes and large 
flooded areas along the southern border with Iran. Mountains rise along the northern borders with Iran and Turkey. It has 36 miles of coastline ${ }^{9}$.

Sur la page consacrée aux cartes, on peut accéder aux cartes à échelle nationale suivantes: population répartie entre Sunnites et Chiites, champs pétroliers et palaces de Saddam Hussein, ainsi qu'une "carte régionale » à plus petite échelle, centrée sur l'Irak et qui s'étend de la Grèce au Turkménistan et à l'Arabie Saoudite. On peut définir ces cartes comme des cartes de cadrage. Le bandeau de défilement propose également des cartes présentant les principales forces militaires en place (bases militaires coalisées et irakiennes par armes) ainsi que les sites de dépôt d'armes (chimiques, biologiques et nucléaires) et une carte régionale précisant la portée des missiles irakiens (figure 4). Deux figurés sont utilisés : le triangle pour localiser les principaux sites et le cercle pour la portée des missiles. Ces dernières cartes font plus précisément référence à un conflit armé potentiel, en présentant l'arsenal irakien ${ }^{10}$, mais elles demeurent des cartes de localisation, rappelant davantage la mission de désarmement confiée aux inspecteurs de l'ONU dans le cadre d'une résolution pacifique du conflit.

Les cartes proposées par USA Today (Interactive war maps) se placent résolument dans un autre registre (figures 5 à 8 , hors-texte). Le quotidien les définit comme des «cartes interactives de la guerre 》 (interactive war maps), là où $\mathrm{CNN}$ ne parlait que de « cartes » (maps). Le bleu profond (intense) n'a que peu à voir avec la réalité d'un terrain désertique chaud. Il s'agit de la couleur de référence du magazine, qui rappelle celle du drapeau national. Le site internet propose une série de cartes établies quotidiennement pour suivre la progression du conflit. Ces cartes n'ont en réalité rien d'interactif, au sens où elles ne permettent en aucun cas d'établir un mode conversationnel. Elles sont fournies telles quelles, sont téléchargeables, mais non enregistrables et non modifiables. Le site ouvre sur la carte du jour et propose les cartes précédemment archivées. Il s'agit indéniablement de cartes d'avancée des troupes à visée performative.

De la lecture géographique de ces cartes, ressortent plusieurs éléments d'analyse. Si l'on suit le déroulement journalier, on note un effet volontaire

9. « L'Irak, avec 168927 miles carrés (437 521 kilomètres carrés) est légèrement plus grande que la Californie. Le pays est principalement constitué de plaines désertiques mais présente également de grandes roselières et zones inondées le long de la frontière méridionale avec l'Iran. Une chaine de montagnes borde la frontière septentrionale avec l'Iran et la Turquie. Il possède 36 miles de côtes ».

10. Rappelons que les médias, et le gouvernement américain, ont largement diffusé l'idée que l'Irak possédait la « quatrième armée du monde » lors de la première guerre du Golfe en 1991. 


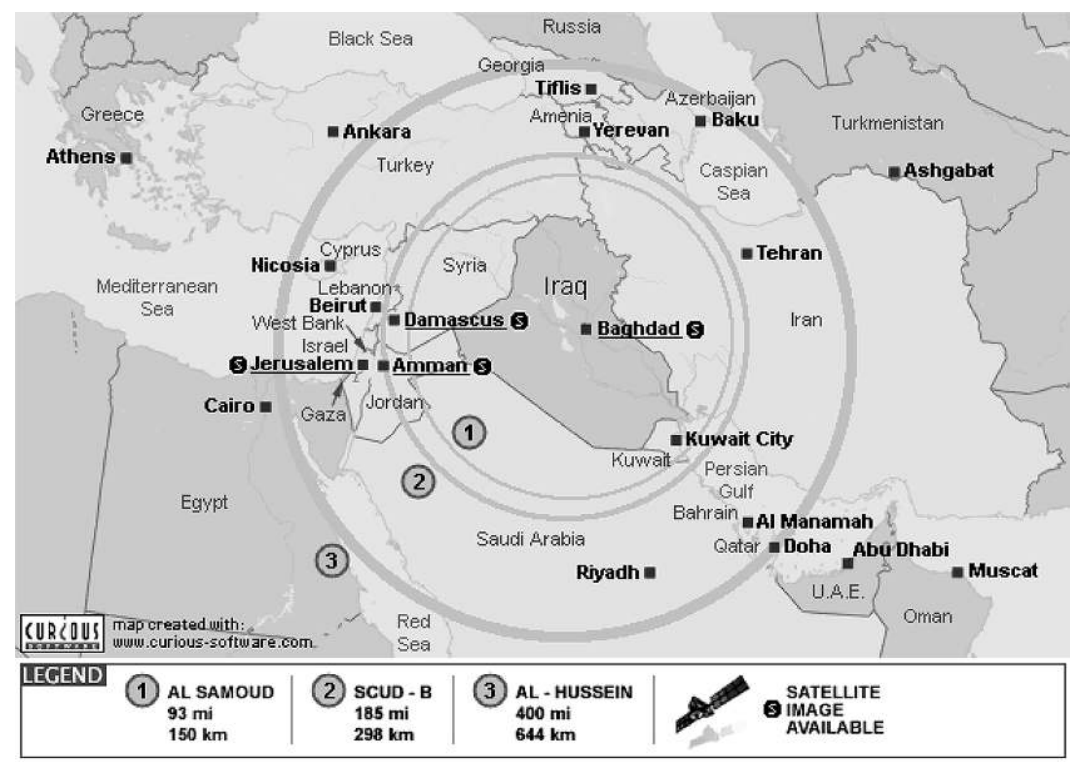

Figure 4

Une carte stratégique ? : la portée des missiles irakiens (source : CNN, avril 2003) "During the Persian Gulf War, Iraqi forces fired Scud missiles into Saudi Arabia and Israel in an effort to disrupt the U.S.-led coalition. After the war, the U.N. limited the Iraqi missiles' range to 93 miles $(150 \mathrm{~km})$. In February 2003, U.N. inspectors said some missile systems exceeded that limit, a charge Iraq downplayed» («Pendant la Guerre du Golfe, les forces irakiennes ont envoyé des missiles Scud sur l'Arabie Saoudite et sur Israël dans le but de rompre la coalition américaine. Après la guerre, l'ONU a limité la portée des missiles irakiens à 93 miles $(150 \mathrm{~km})$. En février 2003, les inspecteurs de l'ONU ont signalé que le système de certains missiles dépassaient cette portée, une accusation que l'Irak a minimisée »).

de zoom sur la capitale irakienne, Bagdad comme la citadelle à prendre dès le premier jour de l'offensive (figure 5). Il n'est alors question que d'attaques de missiles Tomahawk lancés de la mer Rouge (au sud-ouest) et du golfe Persique (au sud-est) et fondant sur Bagdad. La carte est simplifiée à l'extrême pour renforcer l'impression d'efficacité des frappes « chirurgicales ». La délimitation des zones d'interdiction de survol aérien crée un effet de rétrécissement du territoire irakien. Ni échelle ni légende pour préciser ce que signifient les trois points rouges ciblés, ni la distinction entre flèches rouges et lignes noires. On prévoit alors une attaque éclair. À partir du cinquième jour, deux cartes sont proposées, partageant le pays en deux sous-régions (Nord et Sud). Cette évolution correspond à l'entrée en scène du problème 
kurde et au refus de la Turquie d'ouvrir ses frontières. Les cartes centrées sur le Sud (figure 6) offrent alors pendant plusieurs jours la vision d'une armée progressant rapidement sur le terrain. L'apparition d'une échelle graphique renforce cette impression. À partir du quatorzième jour de combat, une nouvelle carte apparait centrée sur la région de Bagdad (figure 7). Deux jours après, à partir de la prise de l'aéroport international à l'ouest de l'agglomération, la carte entre dans la ville en même temps que les chars Abraham (figure 8).

À travers les quatre cartes retenues, on peut se livrer à un exercice de sémiologie graphique. Les couleurs primaires utilisées accentuent la vigueur de l'attaque. Bleu, rouge, blanc, les couleurs du Star \& Stripes. Les éléments de cadrage physique sont réduits à la portion congrue : aucune indication de relief, Tigre et Euphrate en arrière-plan. Le réseau viaire de Bagdad figure en arrière-plan grisé, largement masqué par la prise en tenailles de la capitale et les frappes ponctuelles sur les emblèmes du pouvoir irakien (figure 8). Les manœuvres militaires se déclinent en points et flèches. La flèche, puis le faisceau de flèches rouges, symbolisent l'avancée des troupes terrestres qui se propagent dans le désert à partir du débarquement d'Oum Ksar. Ce déploiement tentaculaire peut être compris comme une figure de la supériorité numérique des forces coalisées. Les points représentent les villes prises ou les passages stratégiques contrôlés (pont sur l'Euphrate, par exemple). Mais les villes restent à l'état de points. On assiste à une géométrisation du réel, par là même déshumanisé, à une fonctionnalisation de l'espace. La carte figure un jeu de pions, tournant volontairement le dos à la complexité historique de la région. On ne cherche pas à savoir si la prise est effective, encore moins si la zone est sécurisée. Simples balises ou sémaphores sur le chemin de Bagdad... qui témoigne d'une résistance à partir du septième jour (figure 6). La couleur orangée retenue pour figurer cette résistance laisse présager une contre-offensive bien peu vigoureuse. La carte de la région de Bagdad au quatorzième jour des combats (figure 7) nous renvoie à une représentation classique des cartes de champs de bataille sur laquelle on figure par de petits drapeaux la localisation des armées, tel le jeu des soldats de plomb de notre enfance. Si on la regarde dans le détail, on note que bien des villes restent aux mains de l'armée irakienne en retrait de l'avancée des troupes coalisées, mais cela est une autre histoire... La victoire se signera à Bagdad. Dans la mise en scène finale de la bataille de Bagdad (figure 8), qui reprend le schéma classique de la bataille urbaine (Stalingrad, Alger, Sarajevo, ...), les cercles concentriques renforcent l'effet de cible, le cœur névralgique est atteint, les forces terrestres fondent sur la ville. Il est révélateur qu'un seul quartier soit signalé, celui de Saddam City, au nord-est de la ville, comme si 


\section{aus Interactive War Map}

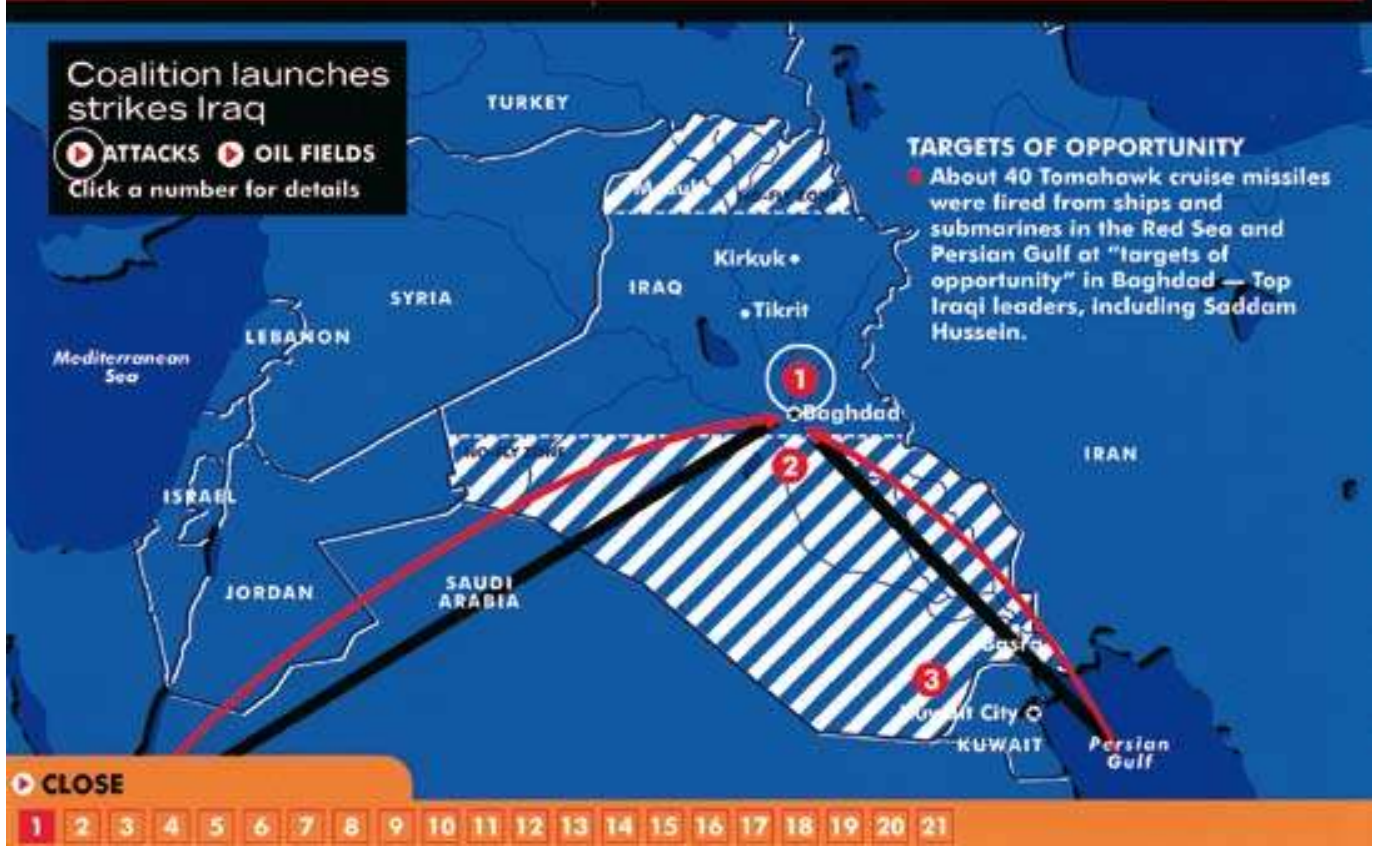

Figure 5

Figure 6

\section{fus Interactive War Map}

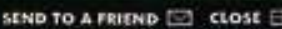

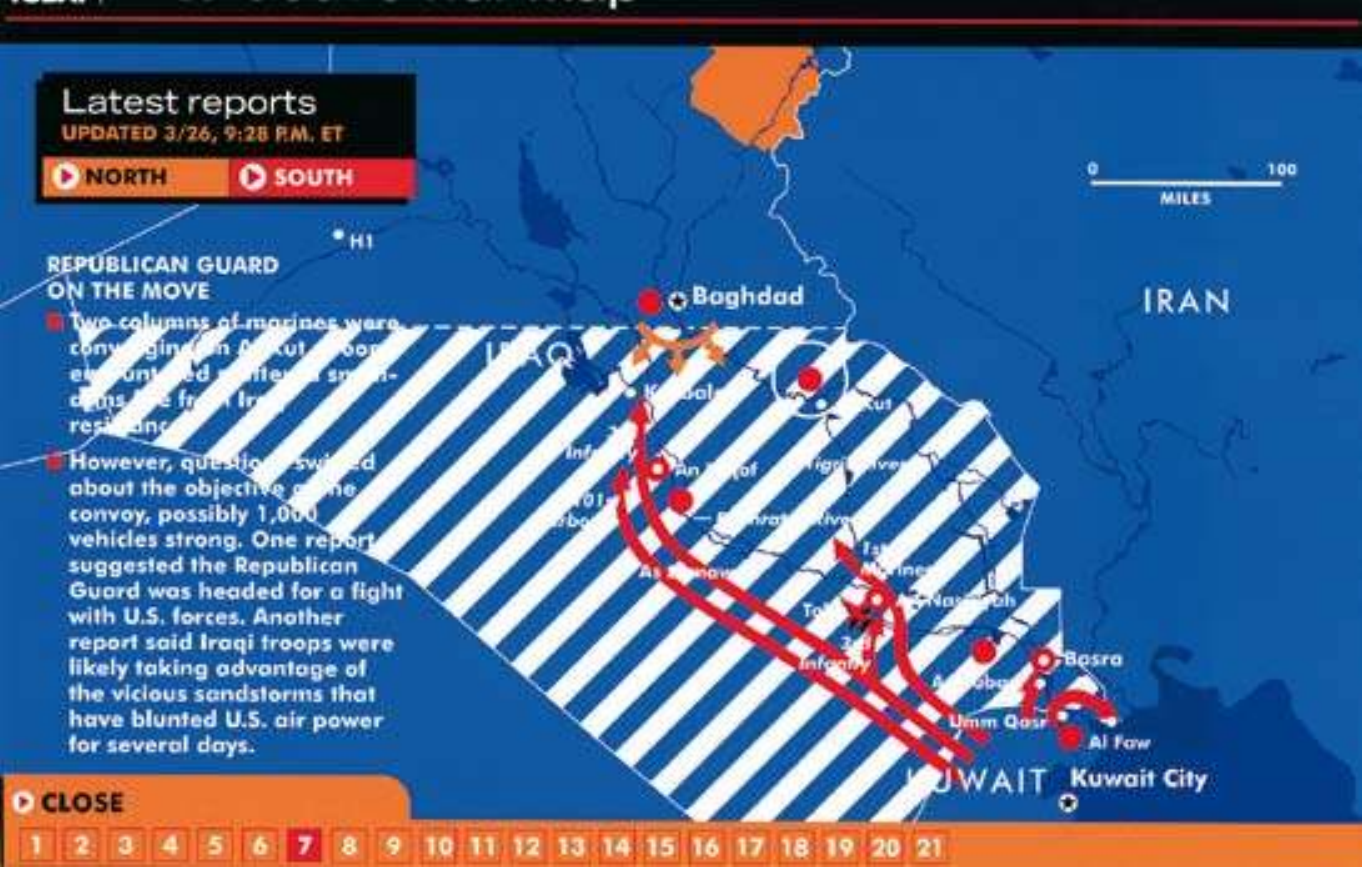


Latest reports

UPDATED 4/2, 11:45 A.M. ET

D BAGHDAD REGION

C NORTHERN IRAQ

DSOUTHERN IRAC

\section{COALITION CROSSES}

TIGRIS NEAR AL KUT

Central Command said

the 1st Marine

Expeditionary Force

crossed the Tigris River

and destroyed the

Baghdad Divison of

the Republican Guard.

The river crossing of $\mathrm{Al}$

Kut opens a key

highway approach to

Baghdad from the

southeest.

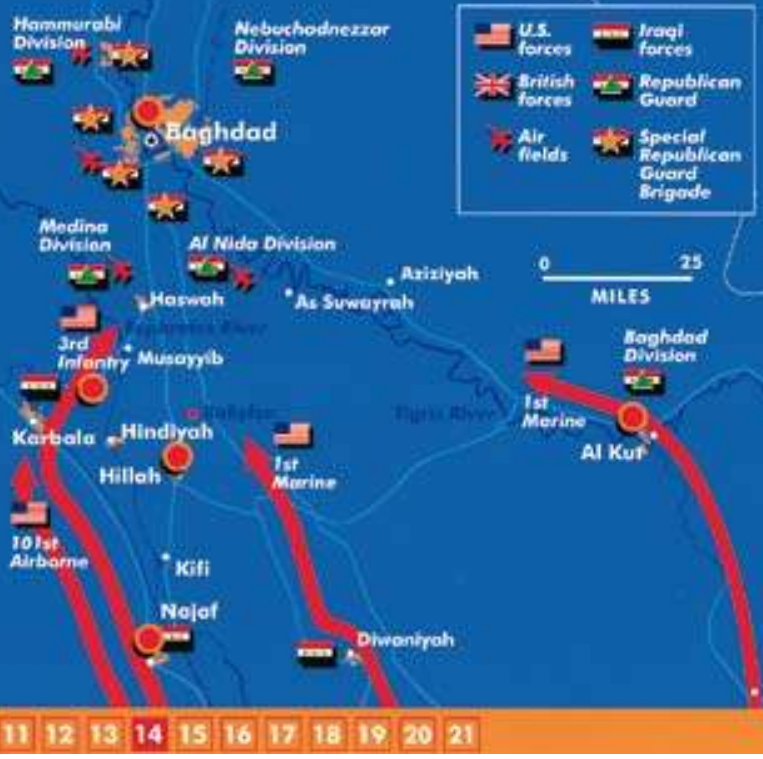

Figure 7

Figure 8

\section{IOMSA Interactive War Map}

SEND TO A FHIND 0 clost $\mathrm{E}$

Latest reports

UPDATED A/8, P:30 PAM. ET

O DOWWITOWN BAGHDAD C BAGHDAD ELCION

O NORTHERN IRAO

o SOUTHERW IRAG

\section{MASSIVE BOMBS}

STRIKE AT LEADERSHIP

AB-1 bomber struck a residence in the upscale Mansur neighborhood with four 2,000-16. bunkerbuster bombs. Intelligence reperts said senior Iraqi officials, possibly. including Saddam and his two sons, were meeting there.

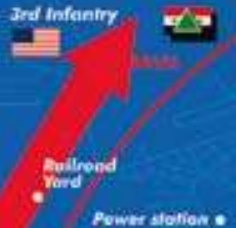

BAGHDAD

Irapi inrelidganctio

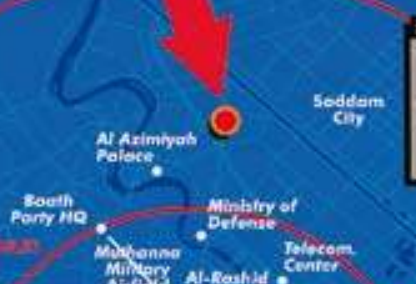

DETAIL AREA

IRAQ

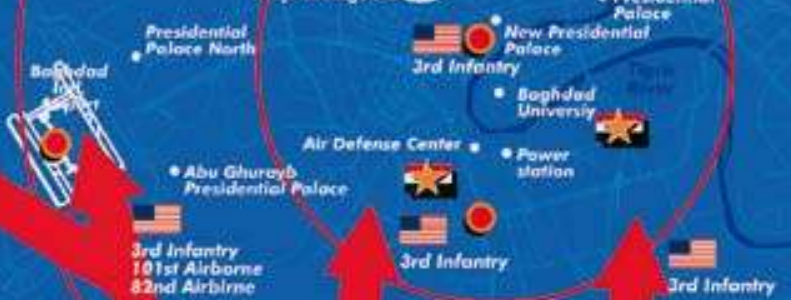


la ville était assimilée de façon bien caricaturale à son dictateur. Le lendemain, vingt et unième jour de combats, toute présence de l'armée irakienne a disparu de la capitale, une dizaine de drapeaux américains flotte sur la carte, ignorant les combats de rue.

Inutile ici de rappeler la force persuasive de la carte. Elle constitue un formidable outil de communication, voire de propagande, dans les relations entretenues entre les états-majors et les médias. Mais il faut se prémunir du revers cynique de la carte qui offre à son lecteur, et à son utilisateur une vision désincarnée de la guerre, comme le rappelle à juste titre B. Harley :

Le savoir cartographique permet la conduite de la guerre par un contrôle à distance, si bien qu'il est plus facile d'envisager des destructions. Non seulement les cartes militaires facilitent la conduite technique de la guerre, mais elles atténuent le sentiment de culpabilité que fait naitre cette conduite : les lignes silencieuses du paysage de papier favorisent l'idée d'un espace socialement vide ${ }^{11}$.

\section{Bibliographie}

BRUNET R., 1987, La carte mode d'emploi, Paris, Fayard-Reclus, 269 p.

BRUNET R., DOLLFUS O., 1994, Mondes nouveaux, t. 1 de la Géographie universelle, Paris, Belin-Reclus, $551 \mathrm{p}$.

BRUNET R., FERRAS R., THERY H., 1992, Les mots de la géographie. Dictionnaire critique, Paris, Reclus, La Documentation française, $470 \mathrm{p}$.

GOULD P., BAILLY A., (éd.), 1995, Le pouvoir des cartes - Brian Harley et la cartographie, Paris, Anthropos, 120 p.

LACOSTE Y., 1976, La géographie, ça sert, d'abord, à faire la guerre, Paris, Maspero, $187 \mathrm{p}$.

MONMONIER M., 1993, Comment faire mentir les cartes - Du mauvais usage de la géographie, Paris, Flammarion, 233 p.

PELLETIER M., 1990, La carte de Cassini - L'extraordinaire aventure de la carte de France, Paris, Presses de l'École nationale des Ponts et Chaussées, 263 p.

11. B. Harley, ouvr. cit., p. 29. 